\title{
WIYN Survey for Carbon Stars in the M 31 and Cetus Dwarf Spheroidal Galaxies: Evolutionary Implications
}

\author{
Daniel Harbeck \\ Department of Astronomy, University of Wisconsin, 475 North Charter Street, Madison, WI \\ 53706 \\ harbeck@astro.wisc.edu \\ John S. Gallagher, III \\ Department of Astronomy, University of Wisconsin, 475 North Charter Street, Madison, WI \\ 53706 \\ jsg@astro.wisc.edu \\ and \\ Eva K. Grebel \\ Astronomical Institute of the University of Basel, Venusstrasse 7, CH-4102 Binningen, \\ Switzerland \\ grebel@astro.unibas.ch
}

\begin{abstract}
We report results of a photometric survey with the WIYN telescope for carbon stars in the M 31 dwarf spheroidal (dSph) companions And III, And V, And VI, and And VII, as well as in the relatively isolated Local Group dSph Cetus. We find three carbonstar candidates in And VII and one carbon star in each And VI and Cetus. Comparing the carbon star content with other Local Group dwarf galaxies, we argue against the presence of substantial intermediate-age stellar populations in the all of the galaxies surveyed with the exception of And VII. We discuss these results in the context of the origin of the Andromeda dSphs and conclude that these are ancient galaxies, most of which ceased star formation long before the main merger events in M31. The M31 dSphs therefore show less diversity in star formation histories than the Galactic dSph companions, or the M $31 \mathrm{dE}$ companions, as illustrated by NGC 147 which was surveyed as a calibration object. All of our dSph targets except And V have candidate carbon stars below the tip of the RGB, which resemble $\mathrm{CH}$ stars found in globular clusters. We estimate that $0.3 \%$ of stars in the dSphs are $\mathrm{CH}$ stars, presumably as a result of $\mathrm{C}$ pollution from a binary companion. Comparisons with $\mathrm{CH}$ star frequencies in globular clusters could constrain the impact of dense environments on the frequency of this form of binary star evolution.
\end{abstract}


Subject headings: Local Group — galaxies: dwarf — galaxies: stellar content — galaxies: evolution — stars: carbon — stars: statistics

\section{Introduction}

The two massive spiral galaxies of the Local group (LG), the Milky Way and Andromeda (M 31) appear to have different histories. While tidal streams associated with minor mergers orbit both spirals (e.g., Ibata, Gilmore, \& Irwin 1995; Ibata et al. 1997, 2001; Ferguson et al. 2002; Newberg et al. 2002, 2003; Majewski et al. 2003; Yanny et al. 2003), the differing levels of interactions in the histories of M 31 and the Milky Way may be reflected in the mean metallicity of their halo stars. M31's halo is more metal-rich than that of the Milky Way, suggesting that mergers have had a substantial impact on M 31 (see Durrell, Harris, \& Pritchet 2001; Brown et al. 2003 and references therein).

We also can ask to what degree the different formation histories of M 31 and the Milky Way are reflected in their systems of satellite galaxies. The dwarf spheroidal (dSph) galaxies of both systems are of special interest because these galaxies might be the unused basic building blocks in hierarchical galaxy formation scenarios (e.g., Bullock, Kravtsov, \& Weinberg 2001), or, alternatively, what should have been larger systems whose structure and evolution was modifed by their proximity to giant spirals (e.g., Mayer at al. 2001). For a more detailed discussion of this subject, see Grebel, Gallagher, \& Harbeck (2003).

While deep photometric studies over wide fields for the Milky Way dSph satellites reach below the oldest main sequence turnoffs (e.g., Hurley-Keller, Mateo, \& Grebel 1999; Harbeck et al. 2001; Carrera et al. 2002; Monelli et al. 2003; Lee et al. 2003), allowing their star formation histories to be studied in detail, the distance of the M 31 dSph subsystem has so far limited existing ground-based photometric studies to the upper red giant branch level (e.g., Armandroff et al. 1993; Armandroff, Davies, \& Jacoby 1998; Grebel \& Guhathakurta 1999) and space-based photometric studies to the horizontal branch level (e.g., Da Costa et al. 2000; Da Costa, Armandroff, \& Caldwell 2002; Harbeck et al. 2001; Karachentsev et al. 2003). Thus, only indirect conclusions can be derived for their star formation history and mean age. Even at this level the M $31 \mathrm{dSphs}$ appear to have unique properties in that Harbeck et al. (2001) found that the M 31 dSphs show a second parameter effect in their horizontal branch (HB) morphologies. After accounting for offsets due to mean metallicities, the M31 dSphs appear to have systematically redder HB morphologies than the Milky Way's dSph companions. The origin of this second parameter effect is not clear, but explanations include a slightly (i.e., 2 Gyr) younger age of the M31 companions compared to their Galactic equivalents (see Da Costa et al. 2000; Harbeck et al. 2001). This has also been suggested for Cetus (Sarajedini et al. (2002)).

In this paper we study the stellar populations in the M $31 \mathrm{dSphs}$ based on properties of stars on the extended asymptotic giant branch (EAGB) containing stars more luminous than the tip 
of the red giant branch (RGB). These include classical EAGB carbon stars (C stars), which trace intermediate-age-to-old (1 to 10 Gyr) stellar populations (Aaronson, Cook, \&Norris 1986). This class of $\mathrm{C}$ star results from thermal pulses and subsequent mixing of freshly synthesized $\mathrm{C}$ into the envelopes of the EAGB stars. The transition on the EAGB from an oxygen rich ( $\mathrm{M}$ star) to $\mathrm{C}$ star therefore depends both on the evolution and initial abundances of the star; lower $\mathrm{O}$ abundances require less $\mathrm{C}$ to make $\mathrm{C} / \mathrm{O}>1$.

A second class of $\mathrm{C}$ star is present even in old globular cluster stellar populations with luminosities below the tip of the RGB. These stars are products of mass transfer from a $\mathrm{C}$ star onto its main sequence binary companion, which subsquently evolves up the RGB (de Kool \& Green 1995; Han et al. 1995). The presence of C stars less luminous than the tip of the RGB is evidence for binary evolution in a stellar population, and is not tied to the age of the population in any simple way. These are spectroscopically identified as $\mathrm{CH}$ stars; for a review of $\mathrm{CH}$ and other types of carbon and related evolved stars, see McClure (1985). The $\mathrm{CH}$ stars in turn are likely to be evolved versions of dwarf carbon (dC) stars (de Kool \& Green 1995), and we discuss implications of the presence of $\mathrm{CH}$ stars in M $31 \mathrm{dSph}$ companions in $\S 3.4$.

While C stars are useful stellar population diagnostics, no systematic wide-field survey for C stars in the M 31 dSph galaxies has been published so far. However, Aaronson et al. (1985) found two EAGB C star candidates in And II, which led them to suggest that And II sustained star formation as an irregular-like galaxy for an extended time. Côté, Oke, \& Cohen (1999) spectroscopically confirmed an additional C star located below the RGB tip luminosity, which they interpreted as being a CH-like star, and probably a product of binary evolution of an undetermined age. However, their color-magnitude diagram (CMD) of And II confirms the presence of an AGB and thus of an intermediate-age stellar population. It also provides a good example of the importance of spectroscopic confirmation in these small galaxies with few EAGB star candidates (see their Fig. 4). In this paper we present a systematic photometric survey using the WIYN 3.5-m telescope ${ }^{1}$ for C star candidates in the Andromeda dSph companions And III, And V, And VI, and And VII ${ }^{2}$, and in the comparatively isolated LG dSph galaxy Cetus. New data for NGC 147 as a calibrator for our survey technique will be presented as well.

\footnotetext{
${ }^{1}$ The WIYN Observatory is a joint facility of the University of Wisconsin-Madison, Indiana University, Yale University, and the National Optical Astronomy Observatories.

${ }^{2}$ Note that And VI and And VII were named Pegasus dSph and Cassiopeia dSph by their discoverers Karachentsev \& Karachentseva (1999) after their parent constellations (see Grebel \& Guhathakurta (1999) for their identification as dSphs and the confirmation of their likely association with M 31). Peg dSph, which was independently discovered by Armandroff, Jacoby, \& Davies (1999), was named And VI by this group. For simplicity, we will use the latter naming convention for the M $31 \mathrm{dSph}$ companions in the following.
} 


\section{Data and Reduction}

We observed the Andromeda companions And III, And V, And VI, and And VII, as well as the Cetus dSph, with the MiniMo Mosaic CCD camera at the $3.5 \mathrm{~m}$ WIYN telescope located at Kitt Peak. We used Johnson V and I filters to obtain temperature and luminosity information for stars in the galaxies in order to construct a color magnitude diagram (CMD), while observations in two narrow-band filters centered on the $\mathrm{TiO}$ and $\mathrm{CN}$ feature at $778 \mathrm{~nm}$ and $808 \mathrm{~nm}$, respectively, were used to identify cool giant stars with enhanced carbon abundance. The use of a CN-TiO filter combination is a well-established method for the reliable identification of C stars (see Cook, Aaronson, \& Norris 1986; Albert, Demers, \&Kunkel 2000; Nowotny et al. 2003). Most of the galaxies were observed during an observing campaign on 2003 September 28 to 2003 October 1.

Since all galaxies are at a similar distance, the exposure times were chosen to be $500 \mathrm{~s}$ in each of the $\mathrm{V}$ and I filter, and $3 \times 500 \mathrm{~s}$ in each of the narrow band filters. There are two exceptions: The exposure times in the V and I band filters for And VII were split into $2 \times 300$ s to avoid CCD blooming of bright stars present in the field of view. The narrow band exposures for And VI were obtained during an earlier observing run at WIYN on 2002 October 20; the exposure times are $2 \times 500$ s per filter. In addition we observed the dwarf elliptical galaxy NGC 147 to validate our ability to identify $\mathrm{C}$ stars. This galaxy is known to have a large number of $\mathrm{C}$ stars (approximately 146 C stars have been found in the work of Nowotny et al. 2003).

Although not always of photometric quality, the data are of superb quality in seeing. The typical seeing in the near infrared narrow-band filters was $0.5^{\prime \prime}$ to $0.6^{\prime \prime}$ and better; the typical seeing in the broad band filters was $0.7^{\prime \prime}$, and never worse then $0.8^{\prime \prime}$. MiniMo at WIYN consists of a mosaic of two CCD chips with a total field of view of $9.6^{\prime} \times 9.6^{\prime}$. The M $31 \mathrm{dSph}$ satellites with typical core radii of order of $1^{\prime}$ to $1.5^{\prime}$ thus conveniently fit onto a single chip, and have been centered on chip \#1 of the mosaic. The more extended galaxies NGC 147 and Cetus were placed at the center of the field of view of MiniMo.

During the 2003 campaign there was a problem with the MiniMo CCD readout electronics, resulting in strong random variations of the CCD's overscan level. Before correcting the raw CCD frames with daily zero calibrations frames, we subtracted the overscan on a line-by-line basis. The variations in the overscan did not completely vanish, leaving residual additional background noise with an RMS of order of 5 counts. Finally the images were corrected for illumination variations using dome flat fields.

For each galaxy, all its images were registered to a common reference coordinate system. The three (or two in the case of And VI) exposures per narrow-band filter were stacked and used for cosmic-ray rejection. In the case of And VII we also stacked the two V and I images. As an example we present a portion of the northern region of NGC 147 in Figure 1. Point spread photometry was 
performed in all filters using the daophot implementation under $\operatorname{IRAF}^{3}$, resulting in photometric catalogs for each galaxy containing instrumental $V, I$, TiO, and $C N$ filter magnitudes.

Since the weather was non-photometric during parts of our observing run, we did not attempt to observe photometric standard stars, and all magnitudes cited in this paper are instrumental magnitudes. In order to identify C stars, we only need differential photometry. For clarity, however, we adopted $I$, and $V-I$ zeropoints for each galaxy to match the magnitude and color of the blue side of the tip of the RGB with observed values (from Sarajedini et al. 2002 for Cetus, from Grebel \& Guhathakurta 1999 for And VII and And VI, from Armandroff, Davies, \& Jacoby 1998 and Armandroff et al. 1993 for And V and And III, respectively; NGC 147 is calibrated according to Nowotny et al. 2003). We calibrated the zeropoint of the $C N-T i O$ color using the assumption that blue stars (i.e., bluer then the tip of the RGB) should have $C N-T i O=0$, since spectra of warmer stars are expected to featureless in this wavelength region (Brewer, Richer, \& Crabtree 1995).

\subsection{Identification of Carbon stars}

The color-magnitude diagram for our test case, NGC 147, is shown in Fig. 3 (left). The intrinsically broad red giant branch (RGB) features prominently as it does in the WFPC2 observations of Han et al. (1997). We also find a prominent population of stars well above the tip of the RGB (TRGB). The right side of the same figure demonstrates the selection of carbon stars from the two-color $(\mathrm{V}-\mathrm{I}$ vs. $\mathrm{CN}-\mathrm{TiO}$ ) diagram: stars with high carbon content (and therefore low $\mathrm{TiO}$ but strong $\mathrm{CN}$ molecule band absorption) stand out in this plot and are selected according to the selection box $(C N-T i O \geq 0.25)$. Stars in this plot with $C N-T i O \geq 0.65 \mathrm{mag}$ are almost always false detections, and are rejected for NGC 147 (see discussion of false detections at the end of this section). Only stars with $I \leq 21 \mathrm{mag}$ are plotted in our two-color diagram to limit this sample to objects with good photometry. Only stars with colors of the RGB and $I \leq 20.7$ mag (Han et al. 1997) qualify as bona-fide C stars; stars fainter than the TRGB might be CH stars and thus are not intermediate age stellar population tracers. C stars selected this way are plotted in the CMD of NGC 147 with filled circles. The substantial number of C star candidates (155) matches our expectation for this galaxy and compares well to the $146 \mathrm{C}$ stars found by Nowotny et al. (2003).

In addition to the photometric selection, we construct the difference of the CCD images observed in the $\mathrm{TiO}$ and $\mathrm{CN}$ filters to cross-identify the candidates on these images. An example of such a difference image is given in Fig. 2, where we zoom into the boxed region of Fig. 1. A visual inspection of the difference image of NGC 147 leads us to estimate a roughly $10 \%$ incompleteness in our C star detections, which is compensated by roughly $10 \%$ false detections. We did not cross-

\footnotetext{
${ }^{3}$ IRAF is distributed by the National Optical Astronomy Observatories, which are operated by the Association of Universities for Research in Astronomy, Inc., under cooperative agreement with the National Science Foundation.
} 
identify each star in our NGC 147 catalog with the difference image, but will do so for the dSph galaxies and will accept only $\mathrm{C}$ stars that appear both in our photometric catalog and stand out as a clear detection in the difference images. The fact that we find a slightly larger number of $\mathrm{C}$ stars in NGC 147 than Nowotny et al. (2003) can be explained by our deeper photometry, better seeing conditions (by at least $0.3^{\prime \prime}$ in the narrow-band filters), and a larger field coverage. A comparison between the catalog of $\mathrm{C}$ stars of Nowotny et al. (2003) indeed reveals that most of the stars are in common with our catalog, with both false detections and misses of order of $10 \%$ in their as well as in our work. We thus increase the number of C stars in NGC 147 to approximately 155, and conclude that our selection efficiency of $\mathrm{C}$ star candidates is comparable to other surveys.

Due to the small absolute number and an improved crowding situation, we slightly loosen the photometric selection criterion for $\mathrm{C}$ stars in the dSphs, by requiring a $\mathrm{CN}-\mathrm{TiO}$ color of $\geq 0.2 \mathrm{mag}$ and a luminosity of the $\mathrm{C}$ star candidates brighter than $I=22 \mathrm{mag}$. This selection will result in some false detections that we identified and removed during the cross-correlation with the difference image. With the small number of $\mathrm{C}$ star candidates per galaxy we can afford to double-check each individual star. Reasons why stars might appear in the photometric catalog

and not in the difference image turned out to be (and are indeed limited to): misidentification of background galaxies, of cosmic ray trails (not all cosmic rays are actually removed), stars affected by stray light or blooming in the vicinity of bright stars in the field of view, blends of two stars, or CCD defects. C star candidates that turned out to be false detections have been marked with open circles in the diagrams. Stars that remain valid C star candidates after cross-identification are plotted with filled circles.

\subsubsection{The Andromeda dSphs}

The CMDs and two-color diagrams of the observed M31 companions are presented in the same manner as for NGC 147 in Figs. 4 to 7. The color of the RGB depends on the metallicity of the galaxies, and we carefully adjusted the $V-I$ color boundary for the $\mathrm{C}$ star selection to avoid confusion with stars bluer than the tip of the RGB. The initial number of photometric detections of $\mathrm{C}$ star candidates that are not cross-identified in the difference image is very different for the four observed galaxies (as indicated by the number of open circles in the diagrams), and turned out to be clearly correlated with the number of (bright) field stars on the CCD frame. The larger the number of bright foreground stars, the larger the number of false detections. After comparing the initial photometric $\mathrm{C}$ star candidates with the difference images, we are left with a smaller set of reliable $\mathrm{C}$ star candidates in the galaxies. These are listed in Table 1.

Depending on whether the bolometric luminosity of a $\mathrm{C}$ star candidate is brighter or clearly dimmer than the tip of the RGB, we classify it as a genuine $\mathrm{C}$ star ("C" in Table 1) or as an evolved dC star (also called "CH" star), respectively. This selection criterion is unique for all but one star in And VII (see Figure 7), which has almost the same luminosity as the TRGB. We classify this star as a $\mathrm{C}$ star candidate. This is justified as technically AGB stars would have 
at least the same bolometric luminosity as the tip of the RGB. If we apply a correction in the form B.C. $=-0.246 \cdot(V-I)$ (Reid \& Mould 1984) this star would clearly satisfy the bolometric luminosity criterion.

The V-and I-band images and the TiO- and CN-band images of And VI were taken during two different campaigns, and due to imperfect overlap of the two observations the total field covered of And VI is limited to a $2.9^{\prime}$-wide stripe (more than twice the core radius of $1.3^{\prime}$, Caldwell 1999). The number of detected $\mathrm{C}$ stars for this galaxy therefore could be incomplete.

The lack of radial velocity information for the $\mathrm{C}$ star candidates prohibits prevents us from verifying their membership in the M31 dSph companion galaxies. Owing to the large angular distance of these galaxies from M31, confusion with M 31 halo C stars seems unlikely. Indeed, all of our $\mathrm{C}$ star candidates are near the central regions of the dSphs, supporting the idea that younger (that is, intermediate-age) populations are primarily found in the central regions of dSphs (see Grebel 1999, 2000; Harbeck et al. 2001). The centralized location of the C stars and their narrow band colors makes it unlikely that we are seeing misidentified Galactic foreground dC stars or backgrund galaxies. In the remainder of this paper we will assume that the $\mathrm{C}$ star candidates are members of the M $31 \mathrm{dSph}$ satellites.

\subsubsection{Cetus}

Cetus was placed on the center of the MiniMo field of view, so there is no photometry available for a central $7^{\prime \prime}$ wide stripe. The CMD and the two-color diagram of Cetus are shown in Figure 8 and Fig. 9 for the MiniMo CCD chip 1 and chip 2, respectively. We can identify three objects with enhanced carbon abundance. One of them is a likely $\mathrm{C}$ star candidate, while two of them appear to be $\mathrm{CH}$ stars or similar objects. However, due to the gap in the MiniMo image we might miss approximately one $(1 \pm 1) \mathrm{C}$ star in Cetus. In good agreement with earlier studies (Whiting, Hau, \& Irwin 1999; Sarajedini et al. 2002) we do not find clear evidence for an EAGB in this galaxy.

\section{Discussion}

We successfully detected high probability candidates both for EAGB C stars and CH stars in four M 31 dSphs surveyed and the Cetus dSph. In his reviews of the C star content of Local Group galaxies, (Groenewegen 1999, 2002) plots absolute magnitude of LG galaxies versus the logarithm of the number of $\mathrm{C}$ stars, carefully corrected for area coverage. We expanded this plot with new data points as shown in Figure 10 using new results from the literature as summarized in Table 2. Absolute $V$-band magnitudes were taken from Grebel, Gallagher, \& Harbeck (2003).

We consider only LG galaxies where surveys for C star candidates using the CN-TiO narrowband filter technique are expected to be reasonably complete. While several $\mathrm{C}$ stars have been 
found in Milky Way dSph galaxies (Aaronson \& Mould 1985; Groenewegen 2002), in some cases these are likely to be $\mathrm{CH}$-stars, and the surveys often have incomplete areal coverage. We therefore include only a few Milky Way dSph satellites in Figure 10. For the SMC and LMC we adopt the DENIS infrared survey results for the number of C stars (Cioni \& Habing 2003).

The galaxies in Figure 10 show the well-known correlation between the absolute V-band luminosity of a galaxy (i.e., an approximate stellar mass) and the total number of $\mathrm{C}$ stars. A useful tool to study the $\mathrm{C}$ star content of a galaxy is $\mathrm{N}_{\mathrm{C}, \mathrm{L}}=\log (\# C)+0.4 \cdot \mathrm{M}_{\mathrm{V}}$, which measures the number of $\mathrm{C}$ stars per unit luminosity, and thus is a measure for the deviation from the $\mathrm{M}_{V}-\log (\# \mathrm{C})$ relation (Aaronson, Olszewski, \& Hodge 1983; Groenewegen 1999). We plot $\mathrm{N}_{\mathrm{C}, \mathrm{L}}$ vs. the mean metallicity of the galaxies in Fig. 10 (right). $\mathrm{N}_{\mathrm{C}, \mathrm{L}}$ scatters between -3 and -4.6 for the galaxies, as previously illustrated by Groenewegen $(1999,2002)$. There is no apparent trend with metallicity, consistent with the predictions of theoretical population models (Mouhcine \& Lançon 2003). These models show that for smoothly evolving stellar populations, $\mathrm{N}_{\mathrm{C}, \mathrm{L}}$ is expected to drop substantially only for super-solar metallicities or systems where the stars are all ancient with ages $\geq 9-10$ Gyr.

We divide the galaxies in this sample into three classes, motivated by their morphology and - where known - their star formation history: (i) "stellar fossil" dSphs, where star formation ceased at very early times, (ii) dwarf irregular galaxies, transition-type galaxies, and dSphs where the period of star formation extends over a substantial fraction of cosmic time, and (iii) the dE companions of M31. Of the Galactic dSphs included in Table 2, a deep HST study of the stellar populations in Leo I revealed that this galaxy was actively forming stars until 1 Gyr ago, with slower ongoing star formation until 300 Myr before the present (Caputo et al. 1997; Gallart et al. 1999). The situation is similar for Fornax, where star formation stopped only 100 to $200 \mathrm{Myr}$ in the past ( Stetson, Hesser, \& Smecker-Hane 1998; Grebel \& Stetson 1999; for recent reviews of the star formation histories of Local Group dwarf galaxies, see Grebel 1999, 2000). These two Galactic dSphs are in our second star forming galaxy category. All of the M 31 dEs have large numbers of intermediate age stars, and low level star formation continues into the present in NGC 185 and NGC 205 (Lee, Freedman, \& Madore 1993; Grebel 1997; Martínez-Delgado, Aparicio, \& Gallart 1999; Cappellari et al. 1999; Davidge 2003). We omit the compact elliptical M32 from our discussion due to the lack of a published survey for C stars.

The different symbols in Fig. 10 refer to the three classes of galaxies we just defined. We can extract a general trend from Fig. 10 (right panel): Quiescent dSph galaxies contain a small number of $\mathrm{C}$ stars per $\mathrm{V}$-band luminosity $\left(\leq 10^{-4.5}\right)$, while those galaxies with active or recent star formation have a higher $\mathrm{C}$ star content $\left(\approx 10^{-3.5}\right)$. The recent star formation in the Fornax $\mathrm{dSph}$ makes this an outstanding galaxy in this plot. The normalized $\mathrm{C}$ star content of the three M 31 dEs (NGC 147, NGC 205, and NGC 185) scatters around $\mathrm{N}_{C, L}=-4$, consistent with their large intermediate-age stellar populations. 


\subsection{Star Formation Histories of the Andromeda dSphs}

The CMDs derived from WFPC2 imaging of And I, And II, And III, And V, And VI, and And VII do not extend much below the horizontal branch. They are therefore sensitive only to potential main sequence stars that have ages of $\leq 1$ Gyr. None of these dSphs show evidence for star formation within this recent time frame (see, e.g., Harbeck et al. 2001; Karachentsev et al. 2003). Furthermore, the CMDs presented by Harbeck et al. (2001) show that red clumps, typical of intermediate age stars, are unlikely to be present in the M $31 \mathrm{dSphs,} \mathrm{and} \mathrm{that} \mathrm{all} \mathrm{the} \mathrm{M} 31 \mathrm{dSphs}$ have pronounced horizontal branches.

\subsection{The $\mathrm{C} / \mathrm{M}$ ratio}

Comparisons of the number of normal EAGB stars, seen as stars with spectral classes of M5 or later, to C stars also is a useful evolutionary diagnostic (see, e.g., Blanco \& McCarthy 1983; Mouhcine \& Lançon 2003; Cioni \& Habing 2003). This ratio depends on both age and metallicity, with the relative number of $\mathrm{C}$ stars peaking for mean ages of $\approx 1 \mathrm{Gyr}$, and in all cases declining for ages greater than $\sim 10$ Gyr. Unfortunately, the relevant ratio of $N_{C} / N_{M 5+}$ is very difficult to derive for the M31 dSphs due to the very small number of $\mathrm{C}$ and M stars on the EAGB and the presence of substantial numbers of red Galactic foreground stars.

An approximate value of $N_{C} / N_{M 5}$ can be obtained from our data by counting all stars redder than $V-I \geq 2.0 \mathrm{mag}$ (to select $M 5+$ spectral classes; Richer, Pritchet, \& Crabtree 1985, Pritchet et al. 1987) and $I<I_{\mathrm{TRGB}}$ (i.e., brighter than the tip of the RGB), or $I>19$. However, we avoid stars near to the saturation limit of the CCD, which are bright foreground stars, and objects whose peculiar colors indicate either that they are not stars or that a problem exists with the data. This approach is most effective when the density of EAGB stars relative to foreground field stars is $>1$, as in NGC 147.

We counted all EAGB $M 5+$ stellar candidates in NGC 147 both in the full field of view (which will lead to an overestimation of $N_{M 5+}$ due to field contamination), and within the central $3^{\prime}$, which may cause us to slightly underestimate $N_{M 5+}$ due to crowding effects. This approach yields

$N_{C} / N_{M 5+}=\frac{159}{1024}=0.16$ and $\frac{111}{575}=0.19$ for the full field and the central region, respectively. Given the expected uncertainty in $N_{C} / N_{M 5+}$ of order of 0.02 we are in excellent agreement with the result of Nowotny et al. (2003), who found $N_{C} / N_{M 5+}=0.15$ for NGC 147.

Since the M 31 dSphs cover a relatively small region of the WIYN MiniMo camera CCD chips, we counted all stars fulfilling the conditions defined above in strips above and below the dSph targets. The resulting estimate of the foreground Galactic star contamination at And VI is too large relative to any population of EAGB $M 5+$ stars to get a meaningful estimate for $N_{M 5+}$. Similarly, we find no M-star candidates in the Cetus dSph, where this method yields $N_{M 5+}=-2 \pm 4$. We also see that since And III and And V have no EAGB C stars, they must have $N_{C} / N_{M 5+}=0$. 
For And VII this method gives $N_{M 5+} \approx 48$. We derive a relative fraction of $\mathrm{C}$ stars of $N_{C} / N_{M 5+}=0.06$ or $\log (C / M) \approx-1.2$. For a galaxy with constant star formation we would expect $\log (C / M) \geq 1$ at $[\mathrm{Fe} / \mathrm{H}] \approx-1.5$ (e.g., Fig. 8 in Mouhcine \& Lançon 2003). If we adopt a correction for the bolometric luminosity (as in Reid \& Mould 1984) to select M stars, we would slightly find $38 \mathrm{M} 5+$ stars despite the fact that we would include even redder stars with I-band luminosities fainter than the tip of the RGB by the selection, which mainly seems to reflect the uncertainty in the background subtraction. Despite the considerable uncertainty in this ratio, we can say there is no indication for the presence of a significant younger intermediate-age ( $<3$ Gyr) stellar population in And VII based on the models of Mouhcine \& Lançon (2003).

The presence of only one carbon star in And VI and Cetus, where we find no significant populations of EAGB $M 5+$-stars might be interpreted as a high $N_{C} / N_{M 5}$ ratio which would be unexpected for a small young stellar population. However, with only one candidate EAGB star in each galaxy, a range of possibilities existis, including the production of an EAGB C star via binary evolution. For example, $\mathrm{C}$ stars should be able to form from blue stragglers. Furthermore, with only one EAGB candidate per galaxy, spectroscopic confirmation of membership is essential.

In summary, only And VII in our sample shows evidence for a substantial EAGB stellar population. In this case the low value of the $N_{C} / N_{M 5+}$ ratio places these stars in the medium range of intermediate stellar ages, namely ages $\geq 3-5$ Gyr.

\subsection{On the origin of the Andromeda dwarf companion galaxies}

We began this investigation with the objective of comparing the dSph companions of M 31 with those of the Milky Way, and of investigating whether the dSphs might be linked to the merger history of M31. Our data are consistent with the M31 dSphs having predominantly old stellar populations, where most star formation occurred at $>10 \mathrm{Gyr}$ in the past. The only clear dSph exception are And II (from Aaronson et al. 1985 work), and And VII, where significant star formation could have continued until 3-5 Gyr ago. Both the survey for EAGB stars presented here and the study of horizontal branch morphologies by Harbeck et al. (2001) are consistent with the view that the majority of M31's dwarf satellites are old systems. In particular, we do not find examples of dSphs with recent star formation activity, like the Fornax or Carina dSph satellites of the Milky Way.

Any connections between the dSphs and stellar halo of M31 remain tenuous. As Ferguson et al. (2002) emphasize, even though And I and And III lie in the projected vicinity of the main M 31 southwestern stellar stream, their low metallicities make dSphs unlikely to have been dominant contributors to the more metal rich components of the M31 halo. A further constraint comes from the Brown et al. (2003) analysis of their spectacularly deep HST CMD of the M31 stellar halo. They find that the significant metal-rich halo component of M31 has an age of 6-8 Gyr, too young to be associated with any of the M $31 \mathrm{dSph}$ companions with the possible exception of 
the distant And VII dSph; see Grebel, Gallagher, \& Harbeck (2003) for deprojected distances and mean metallicities of the M 31 dwarf companions.

In terms of their star-formation histories, M31's dSphs resemble the ancient Galactic dSph companions as epitomized by the Draco and Ursa Minor dSphs. Of these two, Draco appears to be dark-matter dominated (e.g., Odenkirchen et al. 2001), whereas Ursa Minor may be undergoing tidal disruption (e.g., Palma et al. 2003), so Draco probably is the best comparison object. The M 31 dSph companions evidently formed as independent objects that are not directly associated with the recently uncovered indications of interactions with M31, which took place during the galactic "middle ages" some 6-8 Gyr in the past.

However, a possible environmental effect is seen in that only two M 31 dSphs evidently supported extended periods of star formation, fewer than in the population of Galactic dSphs located at similar distances from their spiral host galaxy. That present-day environment cannot be the only agent becomes apparent when considering the similarly quiescent, isolated Cetus dSph (see Grebel, Gallagher, \& Harbeck (2003) for a more extensive discussion). An independent constraint on the importance of dSphs for the accretion history of M 31 could be established by a study of the $[\alpha / \mathrm{Fe}]$ abundances in both M 31 and its companions to see whether halo and dSphs exhibit similar differences as found for the Milky Way by, e.g., Shetrone, Côté, \& Sargent (2001). Unfortunately, because of the distance of M31 and its satellites only global $[\mathrm{Fe} / \mathrm{H}]$ values can be measured spectroscopically to date (Côté, Oke, \& Cohen 1999; Guhathakurta, Reitzel, \& Grebel 2000; Reitzel \& Guhathakurta 2002).

\subsection{On the frequency of $\mathrm{dC} / \mathrm{CH}$ stars}

The standard scenario of "extrinsic" C star formation implies that a main sequence star accretes material through Roche-lobe overflow from its intrinsic EAGB C star binary companion. This mechanism also is responsible for CH-stars (see McClure \& Woodsworth 1990, Han et al. 1995) and we can therefore consider the $\mathrm{dC}$ and $\mathrm{CH}$-like stars to be tracers of similar effects of binary evolution. But what fraction of stars in a stellar population will suffer from pollution severe enough to turn them into C stars? This number depends both on the binary fraction, and the distribution of binary star semi-major axes, metallicity, and age (see, e.g., de Kool \& Green 1995). A "hard" binary system with a orbital separation smaller then a typical AGB radius might disrupt the evolving AGB star before a third dredge-up is able to turn the AGB star into a $\mathrm{C}$ star. Binary-orbit shrinking due to close three-body encounters in dense globular clusters could reduce the specific frequency of dC stars (Côté et al. 1997). Thus the frequency of dC and CH stars is expected to depend also on the environment, and we should find relatively more $\mathrm{dC} / \mathrm{CH}$ stars in low density $\mathrm{dSph}$ galaxies with old stellar populations than in globular clusters.

To estimate the relative number of $\mathrm{CH}$-stars in the uncrowded dSph galaxies, we counted the number of RGB stars brighter than the $I=22 \mathrm{mag}$ limit of our $\mathrm{C}$ star survey. The ratio between 
numbers of CH-stars, which we approximate as evolved dC stars, and RGB stars is an approximate measure of the relative fraction of $\mathrm{dC}$ stars in the stellar populations of these galaxies. However, the small number statistics of the rare C-rich stars introduces substantial uncertainties into this ratio. In Table 3 we present the number of RGB stars and $\mathrm{CH}$ star candidates, as well as the estimated percentage of $\mathrm{CH}$ stars for the galaxies surveyed. We assume that all of our candidates are CH stars in the M 31 dSphs. Spectroscopic follow-up of these stars is essential to confirm their membership by their radial velocities and verify that they are C-rich objects.

To improve the statistics, we simply sum up all of the RGB stars and CH stars to obtain a first estimate of the dSph $\mathrm{CH}$ star frequency. We find $\sim 0.3 \%$ of the dSph's stars to be $\mathrm{CH}$ stars that could have originated from $\mathrm{dC}$ stars which were contaminated during the evolution of a binary stellar system. The frequency of CH stars in globular clusters remains to be established since such stars are very rare (see Côté et al. 1997).

In NGC 147 we count CH stars in the same way as we did for the dSphs between the tip of the RGB and $I \leq 22$, and also count RGB stars in the same luminosity range. This way we derive a slightly higher $\mathrm{CH}$ stars frequency of $0.8 \%$, which might be unreliable since strong crowding might become important for the selection of fainter carbon-rich stars and confusion with genuine $\mathrm{C}$ stars at the bright end of the selection might lead to an overestimation of the number of $\mathrm{CH}$ stars.

\section{Summary}

We present results from a photometric survey for $\mathrm{C}$ stars and related carbon-rich stars in the And III, And V, And VI, And VII, and Cetus dSph galaxies carried out with the WIYN 3.5-m telescope. Similar data also were obtained for the dE galaxy NGC 147, previously surveyed for C stars by Nowotny et al. (2003), as a check on our technique. The small number of C stars found among the M $31 \mathrm{dSph}$ satellite galaxies implies that they contain predominantly old stellar populations. Aside from And II and especially And VII, where star formation may have been active as recently as 3-5 Gyr in the past, the And III, And V, and And VI dSphs have not supported significant star formation within the past $\sim 10$ Gyr.

The stellar populations of the M31 dSphs both are older and more metal-poor than the intermediate-age M 31 stellar halo components. We conclude that the dSph satellites are an early legacy of the time before the prominent M 31 merger events that occurred about 6-8 Gyr in the past. The lack of a connection to the M 31 "middle ages" merger is further supported by the similarities in stellar populations between the M 31 satellites and relatively isolated Cetus dSph, as well as the Galactic dSphs with old populations, such as the Draco or UMi dSphs.

The frequency of C stars in the M $31 \mathrm{dE}$ companion NGC 147 from our data and the recent

study by Nowotny et al. (2003), places it at levels the actively star forming Magellanic Clouds and Fornax or Leo I, Galactic dSphs where star formation continued to within $\sim 1$ Gyr of the present. While the M31 dEs structurally resemble scaled up dSph systems, they have very different star 
formation histories than most of the M 31 dSphs. This serves to emphasize the profound differences in stellar population characteristics among the M $31 \mathrm{dSph}$ satellites. The low luminosity dSphs are predominantly stellar fossils, while the dEs made many of their stars 5-10 Gyr in the past. These $\mathrm{dE}$ galaxies therefore may be associated with some of the more pronounced merger features in M 31 , as others have suggested on the basis of their structures and mean stellar metallicities (Ferguson et al. 2002). The And VII dSph is neither a dE-like object nor a collection of primarily ancient stars, and instead resembles Galactic dSphs which experienced star formation for more than $\sim 5$ Gyr after their formation.

We also found that roughly $0.3 \%$ of the stars in dSphs to be candidate $\mathrm{CH}$ stars, products of binary evolution. Future investigations of $\mathrm{dC} / \mathrm{CH}$ fractions in globular clusters will allow to investigate the impact of a dense environment on binary evolution.

These observations reflect the dedicated efforts of the WIYN Observatory staff, and we thank them for their support. DH gratefully acknowledges support as a McKinney postdoctoral fellow and from the Graduate School at the University of Wisconsin-Madison. JSG acknowledges essential funding from NSF grant AST-9803018 to the University of Wisconsin and expresses his appreciation to Andrew Cole, Ariane Lançon, and Mustapha Mouhcine for discussions regarding the properties and importance of E-AGB stars. EKG and JSG also thank the Swiss National Science Foundation for partial support, and all three authors express their appreciation to the Max-Planck-Gesellschaft for supporting stimulating workshops on dwarf galaxies. This research has made use of NASA's Astrophysics Data System (ADS) and the NASA/IPAC Extragalactic Database (NED) which is operated by the Jet Propulsion Laboratory, California Institute of Technology, under contract with the National Aeronautics and Space Administration. 


\section{REFERENCES}

Aaronson, M., Olszewski, E. W., Hodge, P. W. 1983, ApJ, 267, 271

Aaronson, M., Mould, J. 1985, ApJ, 290, 191

Aaronson, M., Olszewski, E., Gordon, G., Mould, J., \& Suntzeff, N. 1985, ApJ, 296, L7

Aaronson, M., Cook, K. H., Norris, J. 1986, in Spectral evolution of galaxies, ASSL Vol. 122 (Dordrecht, Reidel), p.171

Albert, L., Demers, S., Kunkel, W. E. 2000, AJ, 119, 2780

Armandroff, T. E., Da Costa, G. S., Caldwell, N., \& Seitzer, P. 1993, AJ, 106, 986

Armandroff, T. E., Davies, J. E., \& Jacoby, G. H. 1998, AJ, 116, 2287

Armandroff, T. E., Jacoby, G. H., \& Davies, J. E. 1999, AJ, 118, 1220

Battinelli, P. \& Demers, S. 2000, AJ, 120, 1801

Blanco, V. M. \& McCarthy, M. F. 1983, AJ, 88, 1442

Brewer, J. P., Richer, H. B., \& Crabtree, D. R. 1995, AJ, 109, 2480

Brown, T. M. et al. 2003, ApJ, 529, L17

Bullock, J. S., Kravtsov, A. V., Weinberg. D. H. 2001, ApJ, 548, 33

Caldwell, N. 1999, AJ, 118, 1230

Cappellari, M., Bertola, F., Burstein, D., Buson, L. M., Greggio, L., \& Renzini, A. 1999, ApJ, 515, L17

Caputo, F., Cassisi, S., Castellani, M., Marconi, G., Santolamazza, P. 1997, AJ, 117, 2199

Carrera, R., Aparicio, A., Martínez-Delgado, D., Alonso-García, J. 2002, AJ, 123, 3199

Cioni, M.-R. L. \& Habing, H. J. 2003, A\&A, 402, 133

Cook, K. H., Aaronson, M., Norris, J. 1986, ApJ, 305, 634

Côté, P., Hanes, D. A., McLaughlin, D. E., Bridges, T. J., Hesser, J. E., \& Harris, G. L. H. 1997, ApJ, 476, L15

Côté, P., Oke, J. B., \& Cohen, J. G. 1999, AJ, 118, 1645

Davidge, T. J. 2000, PASP, 112,

Davidge, T. J. 2003, ApJ, 597, 289 
Da Costa, G. S., Armandroff, T. E., Caldwell, N., \& Seitzer, P. 2000, AJ, 119, 705

Da Costa, G. S., Armandroff, T. E., \& Caldwell, N. 2002, AJ, 124, 332

Demers, S. \& Battinelli, P. 2002, AJ, 123, 238

Demers, S., Battinelli, P., \& Letarte, B. 2003, AJ, 125, 3037

Da Costa, G. S., Armandroff, T. E., Caldwell, N., \& Seitzer, P. 2000, AJ, 119, 705

Durrell, P. R., Harris, W. E., Pritchet, C. J. 2001, AJ, 121, 2557

Ferguson, A. M. N., Irwin, M. J., Ibata, R. A., Lewis, G. F., Tanvir, N. R. 2002, AJ, 124, 1452

Gallart, C., Freedman, W. L., Aparicio, A., Bertelli, G., \& Chiosi, C. 1999, AJ, 118, 2245

Grebel, E. K. 1997, Reviews of Modern Astronomy, 10, 29

Grebel, E. K. 1999, in The Stellar Content of the Local Group, IAU Symposium 192, eds. P. Whitelock \& R. Cannon (San Francisco: ASP), 17

Grebel, E. K. 2000, in Star Formation from the Small to the Large Scale, ESA SP-445, eds. F. Favata, A.A. Kaas, \& A. Wilson (Noordwijk: ESA), 87

Grebel, E. K. \& Guhathakurta, P. 1999, ApJ, 511, L101

Grebel, E. K. \& Stetson, P. B. 1999, in The Stellar Content of the Local Group, IAU Symposium 192, eds. P. Whitelock \& R. Cannon (San Francisco: ASP),165

Grebel, E. K., Gallagher, J. S., \& Harbeck, D. 2003, AJ, 125, 1926

Groenewegen, M. A. T. 1999, in Asymptotic Giant Branch Stars, IAU Symp. 191, eds. T. Le Bertre, A. Lébre, \& C. Waelkens (San Francisco: ASP), 535

Groenewegen, M. A. T. 2002, in Chemical Evolution of Dwarf Galaxies, Ringberg Workshop, astro$\mathrm{ph} / 0208449$

de Kool, M. \& Green, P. J. 1995, ApJ, 449, 236

Guhathakurta, P., Reitzel, D. B., \& Grebel, E. K. 2000, Proc. SPIE, 4005, 168

Hane, Z., Eggleton, P. P., Podsiadowski, P., Tout, C. A. 1995, MNRAS, 277, 1443

Han, M., Hoessel, J. G., Gallagher, J. S., Holtzman, J., Stetson, P. B. 1997, AJ, 113, 1001

Harbeck, D. et al. 2001, AJ, 122, 3092

Hurley-Keller, D., Mateo, M., \& Grebel, E. K. 1999, ApJ, 523, L25

Ibata, R. A., Gilmore, G., \& Irwin, M. J. 1995, MNRAS, 277, 781 
Ibata, R. A., Wyse, R. F. G., Gilmore, G., Irwin, M. J., \& Suntzeff, N. B. 1997, AJ, 113, 634

Ibata, R. Irwin, M., Lewis, G., Ferguson, A. M. N., Tanvir, N. 2001, Nature, 412, 49

Karachentsev, I. D. \& Karachentseva, V. E. 1999, A\&A, 341, 355

Karachentsev, I. D., Sharina, M. E., Dolphin, A. E., \& Grebel, E. K. 2003, A\&A, 408, 111

Lee, M. G., Freedman, W. L., \& Madore, B. F. 1993, AJ, 106, 964

Lee, M.G., et al. 2003, AJ, 126, 2840

Majewski, S.R., Skrutskie, M.F., Weinberg, M.D., \& Ostheimer, J.C. 2003, ApJ, 599, 1082

Margon, B. et al. 2002, AJ, 124, 1651

Martínez-Delgado, D., Aparicio, A., \& Gallart, C. 1999, AJ, 118, 2229

Mayer, L. et al. 2001, ApJ, 547, L123

McClure, R. D. 1985, JRASC, 79, 277

McClure, R. D., Woodsworth, A. W. 1990, ApJ, 352, 709

Monelli, M. et al. 2003, AJ, 126, 218

Mouhcine, M. \& Lançon, A. 2003, MNRAS, 338, 572

Newberg, H. J. et al. 2002, ApJ, 569, 245

Newberg, H. J. et al. 2003, ApJ, 596, L191

Nowotny, W., Kerschbaum, F., Olofsson, H., \& Schwarz, H. E. 2003, A\&A, 403, 93

Odenkirchen, M. et al. 2001, AJ, 122, 2538

Palma, C., Majewski, S. R., Siegel, M. H., Patterson, R. J., Ostheimer, J. C., \& Link, R. 2003, AJ, 125,1352

Pritchet, C. J., Schade, D., Richer, H. B., Crabtree, D., \& Yee, H. K. C. 1987, ApJ, 323, 79

Reid, N. \& Mould, J. 1984, ApJ, 284, 98

Reitzel, D. B. \& Guhathakurta, P. 2002, AJ, 124, 234

Richer, H. B., Pritchet, C. J., \& Crabtree, D. R. 1985, ApJ, 298, 240

Sarajedini, A. et al. 2002, ApJ, 567, 915

Shetrone, M. D., Côté, P., \& Sargent, W. L. W. 2001, ApJ, 548, 592 
Stetson, P. B., Hesser, J. E., \& Smecker-Hane, T. A. 1998, PASP, 110, 533

Whitelock, P., Menzies, J., Irwin, M., \& Feast, M. 1999, IAU Symposium, 192, 136

Whiting, A. B., Hau, G. K. T., \& Irwin, M. 1999, AJ, 118, 2767

Yanny, B. et al. 2003, ApJ, 588, 824 
harbeck_f01.jpg

Fig. $1 .-3.5 \times 3.5^{\prime}$ cut-out of the NGC $147 \mathrm{CN}$-filter image (seeing $\leq 0.6$ "). The box indicates the zoom area of Fig. 2, where we show an example of a difference image. East is right, north down. 
Fig. 2.-Zoom $(\approx 45 \times 45 ")$ into a difference $T i O-C N$ image in the region boxed in Fig. 1. C stars with weak $\mathrm{TiO}$ absorption and strong $\mathrm{CN}$ absorption appear as bright objects (encircled), while normal stars just subtract out. The boxed region shows an example of imperfect image subtraction of brighter stars; these objects do not appear in the C star catalog thanks to a cut in the candidates luminosity. 


harbeck_f03.jpg harbeck_f04.jpg

Fig. 3.- Color-magnitude diagram (CMD; left) and two-color diagram (right) of NGC 147; all magnitudes are instrumental, but zeropoint corrected as described in the text. The two-color plot shows the broad-band colors of stars in NGC 147 (i.e., a temperature) versus the relative CN absorption strength as measured by the $\mathrm{CN}-\mathrm{TiO}$ filter colors; only stars brighter than $I=22 \mathrm{mag}$ are plotted (indicated by a line in the CMD, left). Red stars with strong CN absorption falling into the selection box are identified as Carbon stars and are clearly marked with filled circles in both plots. 


\begin{tabular}{|l|l|}
\hline harbeck_f05.jpg harbeck_f06.jpg \\
\hline
\end{tabular}

Fig. 4. - Same as Fig. 3, but showing the CMD and two-color diagram for the And III dSph galaxy. C-star candidates that could be cross-identified on the TiO-CN difference image are plotted with filled circles, false detections with open circles. 


\begin{tabular}{|l|l|}
\hline harbeck_f07.jpg harbeck_f08.jpg \\
\hline
\end{tabular}

Fig. 5.- Same as Fig. 4 for the And V dSph galaxy. 


\begin{tabular}{|l|l|}
\hline harbeck_f09.jpg harbeck_f10.jpg \\
\hline
\end{tabular}

Fig. 6.- Same as Fig. 4 for the And VI dSph galaxy. 


\begin{tabular}{|l|l|}
\hline harbeck_f11.jpg harbeck_f12.jpg \\
\hline
\end{tabular}

Fig. 7.- Same as Fig. 4 for the And VII dSph galaxy. 


\begin{tabular}{|l|l|}
\hline harbeck_f13.jpg harbeck_f14.jpg \\
\hline
\end{tabular}

Fig. 8. - Same as Fig. 4 for stars of the Cetus dSph that fall on Chip \#1.

\begin{tabular}{|l|}
\hline harbeck_f15.jpg harbeck_f16.jpg \\
\hline
\end{tabular}

Fig. 9.- Same as Fig. 4, but for stars of the Cetus dSph that fall on Chip \#2. 


\begin{tabular}{|l|l|}
\hline harbeck_f17.jpg harbeck_f18.jpg \\
\hline
\end{tabular}

Fig. 10.- Left: The relation between the number of $\mathrm{C}$ stars and the luminosity of Local Group dwarf galaxies. Right: The same as left, but with the mean metallicity on the X-axis. 
Table 1. C Stars in Local Group dSphs

\begin{tabular}{|c|c|c|c|c|c|}
\hline Galaxy & Id & $\mathrm{Ra}^{\mathrm{a}}$ & $\operatorname{Dec}^{\mathrm{a}}$ & $\mathrm{M}_{\mathrm{I}}^{\mathrm{b}}$ & $\mathrm{dC} / \mathrm{C}^{\mathrm{c}}$ \\
\hline And III & and3-2538 & $0: 35: 35.19$ & $36: 29: 00.3$ & 21.0 & $\mathrm{dC}$ \\
\hline And V & $\ldots$ & $\ldots$ & $\ldots$ & $\ldots$ & $\ldots$ \\
\hline \multirow[t]{2}{*}{ And VI } & and6-6618 & 23:51:46.63 & $24: 34: 59.3$ & 20.2 & $\mathrm{C}$ \\
\hline & and6-10813 & 23:51:58.96 & $24: 34: 46.9$ & 21.8 & $\mathrm{CH}$ \\
\hline \multirow[t]{5}{*}{ And VII } & and7-3727 & $23: 26: 30.78$ & $50: 40: 50.6$ & 20.7 & $\mathrm{C}$ \\
\hline & and $7-5653$ & $23: 26: 37.54$ & $50: 41: 28.5$ & 21.0 & $\mathrm{C}$ \\
\hline & and7-6111 & 23:26:39.56 & $50: 39: 49.1$ & 20.9 & $\mathrm{C}$ \\
\hline & and 7-4002 & $23: 26: 31.70$ & $50: 40: 57.6$ & 21.3 & $\mathrm{CH}$ \\
\hline & and $7-4447$ & 23:26:33.20 & 50:41:08.6 & 21.4 & $\mathrm{CH}$ \\
\hline \multirow[t]{3}{*}{ Cetus } & cetus-II705 & $00: 26: 12.99$ & $-11: 01: 20.2$ & 20.6 & $\mathrm{C}$ \\
\hline & cetus-II736 & 00:26:13.64 & $-11: 02: 16.7$ & 21.3 & $\mathrm{CH}$ \\
\hline & cetus-I3089 & $00: 26: 13.17$ & $-11: 03: 59.7$ & 21.1 & $\mathrm{CH}$ \\
\hline \multicolumn{6}{|c|}{${ }^{\mathrm{a}} \mathrm{J} 2000.0$, based on USNO2.0 catalog } \\
\hline \multicolumn{6}{|c|}{ binstrumental magnitudes, but zero point corrected in I. } \\
\hline \multicolumn{6}{|c|}{$\begin{array}{l}{ }^{\mathrm{c}} \text { Classification into genuine Carbon star }(\mathrm{C}) \text { and evolved dwarf } \\
\text { Carbon star }(\mathrm{CH})\end{array}$} \\
\hline
\end{tabular}


Table 2. C stars in LG dwarf galaxies

\begin{tabular}{lccccl}
\hline \hline Galaxy & $\mathrm{M}_{V}{ }^{1}$ & $\log \# \mathrm{C}$ & {$[\mathrm{Fe} / \mathrm{H}]^{1}$} & Type $^{1}$ & Source $^{2}$ \\
\hline Phoenix & -9.8 & 0.3 & -1.9 & 2 & 1 \\
DDO 210 & -10.9 & 0.45 & -1.9 & 2 & 1 \\
Leo I & -11.9 & 1.2 & -1.4 & 2 & 1 \\
Peg DIG & -12.9 & 1.6 & -2.0 & 2 & 1 \\
Fornax & -13.1 & 2.0 & -1.2 & 2 & 1 \\
IC 1613 & -15.3 & 2.3 & -1.4 & 2 & 1 \\
And II & -11.8 & 0.3 & -11.5 & 1 & 9,10 \\
And III & -10.2 & $\ldots$ & -1.7 & 1 & 2 \\
And V & -9.1 & $\ldots$ & -1.9 & 1 & 2 \\
And VI & -11.3 & 0 & -1.7 & 1 & 2 \\
And VII & -12.0 & 0.47 & -1.5 & 1 & 2 \\
Cetus & -10.1 & 0 & -1.7 & 1 & 2 \\
NGC 147 & -15.1 & 2.17 & -1.1 & 3 & 2 \\
NGC 185 & -15.6 & 2.19 & -0.8 & 3 & 3 \\
Sag DIG & -12.0 & 1.2 & -2.3 & 2 & 4 \\
NGC 205 & -16.4 & 2.7 & -0.5 & 3 & 5 \\
Sgr & -15.0 & 1.41 & -0.5 & 1 & 6 \\
SMC & -17.1 & 3.35 & -1.2 & 2 & 7 \\
LMC & -18.5 & 4.04 & -0.6 & 2 & 7 \\
M 32 & -16.5 & $\ldots$ & -1.1 & 3 & 8 \\
\hline & & & & & \\
\hline
\end{tabular}

${ }^{1}$ Taken from Grebel, Gallagher, \& Harbeck (2003); Type: 1 - dSph, 2 - dIrr/transition type/dSph with recent star formation, $3-\mathrm{dE}$

21 - Battinelli \& Demers (2000); 2 - this work; 3 Nowotny et al. (2003); 4 - Demers \& Battinelli (2002); 5 - Demers, Battinelli, \& Letarte (2003); 6 - Whitelock et al. (1999); ; 7 - Cioni \& Habing (2003); 8 - Davidge (2000); 9 - Aaronson et al. (1985); 10 - Côté, Oke, \& Cohen (1999) 
Table 3. On the dC star frequency

\begin{tabular}{lccc}
\hline \hline Galaxy & \#RGB stars & \#dC stars & $\frac{\text { \#RGBstars }}{\text { \#dCstars }}[\%]$ \\
\hline And III & 439 & 1 & 0.23 \\
And V & 187 & 0 & 0.00 \\
And VI & 228 & 1 & 0.44 \\
And VII & 1247 & 2 & 0.16 \\
Cetus & 319 & 2 & 0.62 \\
\hline All & 2420 & 6 & 0.25 \\
\hline
\end{tabular}


This figure "harbeck_f01.jpg" is available in "jpg" format from: http://arxiv.org/ps/astro-ph/0401595v1 
This figure "harbeck_f02.jpg" is available in "jpg" format from: http://arxiv.org/ps/astro-ph/0401595v1 
This figure "harbeck_f03.jpg" is available in "jpg" format from: http://arxiv.org/ps/astro-ph/0401595v1 
This figure "harbeck_f04.jpg" is available in "jpg" format from: http://arxiv.org/ps/astro-ph/0401595v1 
This figure "harbeck_f05.jpg" is available in "jpg" format from: http://arxiv.org/ps/astro-ph/0401595v1 
This figure "harbeck_f06.jpg" is available in "jpg" format from: http://arxiv.org/ps/astro-ph/0401595v1 
This figure "harbeck_f07.jpg" is available in "jpg" format from: http://arxiv.org/ps/astro-ph/0401595v1 
This figure "harbeck_f08.jpg" is available in "jpg" format from: http://arxiv.org/ps/astro-ph/0401595v1 
This figure "harbeck_f09.jpg" is available in "jpg" format from: http://arxiv.org/ps/astro-ph/0401595v1 
This figure "harbeck_f10.jpg" is available in "jpg" format from: http://arxiv.org/ps/astro-ph/0401595v1 
This figure "harbeck_f11.jpg" is available in "jpg" format from: http://arxiv.org/ps/astro-ph/0401595v1 
This figure "harbeck_f12.jpg" is available in "jpg" format from: http://arxiv.org/ps/astro-ph/0401595v1 
This figure "harbeck_f13.jpg" is available in "jpg" format from: http://arxiv.org/ps/astro-ph/0401595v1 
This figure "harbeck_f14.jpg" is available in "jpg" format from: http://arxiv.org/ps/astro-ph/0401595v1 
This figure "harbeck_f15.jpg" is available in "jpg" format from: http://arxiv.org/ps/astro-ph/0401595v1 
This figure "harbeck_f16.jpg" is available in "jpg" format from: http://arxiv.org/ps/astro-ph/0401595v1 
This figure "harbeck_f17.jpg" is available in "jpg" format from: http://arxiv.org/ps/astro-ph/0401595v1 
This figure "harbeck_f18.jpg" is available in "jpg" format from: http://arxiv.org/ps/astro-ph/0401595v1 